\title{
A Young Man with Red Eyes, Polymorphous Rash, and Mucositis-an On-the-Spot Diagnosis
}

\author{
Aldo Barajas-Ochoa, $\mathrm{MD}^{\top} \odot$, Nora Montealegre, $\mathrm{MD}^{2}$, and Arturo Perez-Peralta, $\mathrm{MD}^{\top}$ \\ 'Department of Medicine, Rutgers New Jersey Medical School, Newark, NJ, USA; ²Department of Neurology, Rutgers New Jersey Medical School, \\ Newark, NJ, USA.
}

J Gen Intern Med 35(4):1309-10

DOI: $10.1007 / \mathrm{s} 11606-020-05663-y$

(c) Society of General Internal Medicine 2020

A previously healthy 18 -year-old man presented with malaise, fever, and rash for 5 days. His only recent medication was amoxicillin for otitis media 2 weeks before symptom onset. Examination revealed conjunctival hyperemia, polymorphous rash, and labial, oral, and nasal mucositis (Figs. 1 and 2). Laboratory testing and a chest radiograph were unremarkable. Bacterial blood cultures, HIV testing, Mycoplasma pneumonia testing, and PCR for herpes simplex virus were negative. He was diagnosed with Stevens-Johnson syndrome (SJS) due to amoxicillin and his symptoms resolved after 3 weeks.

SJS and toxic epidermal necrolysis (TEN) represent a spectrum of severe mucocutaneous reactions characterized by skin necrosis and sloughing. In SJS, $<10 \%$ total body surface area (TBSA) is affected; TEN affects $>30 \%$ TBSA and SJS/TEN overlap affects $10-30 \%$ TBSA ${ }^{1-3}$. Medications trigger $66-$ $75 \%$ cases, and the most common offending agents are sulfonamides, allopurinol, phenytoin, and NSAIDs ${ }^{1-3}$. A prodrome of fever and influenza-like symptoms occurs 4-28 days after exposure to the offending agent ${ }^{3}$. Atypical target lesions and violaceous macules appear within 1-3 days of prodrome onset, with widespread blister formation that progresses to skin sloughing. Oral mucositis occurs in most cases, and ocular involvement occurs in $80 \%$ of cases with varying severity $1,3,4$. Treatment consists of wound care, fluid/ nutritional support, pain control, and removal of offending agents. Ocular involvement requires topical corticosteroids and antibiotics ${ }^{3}$.

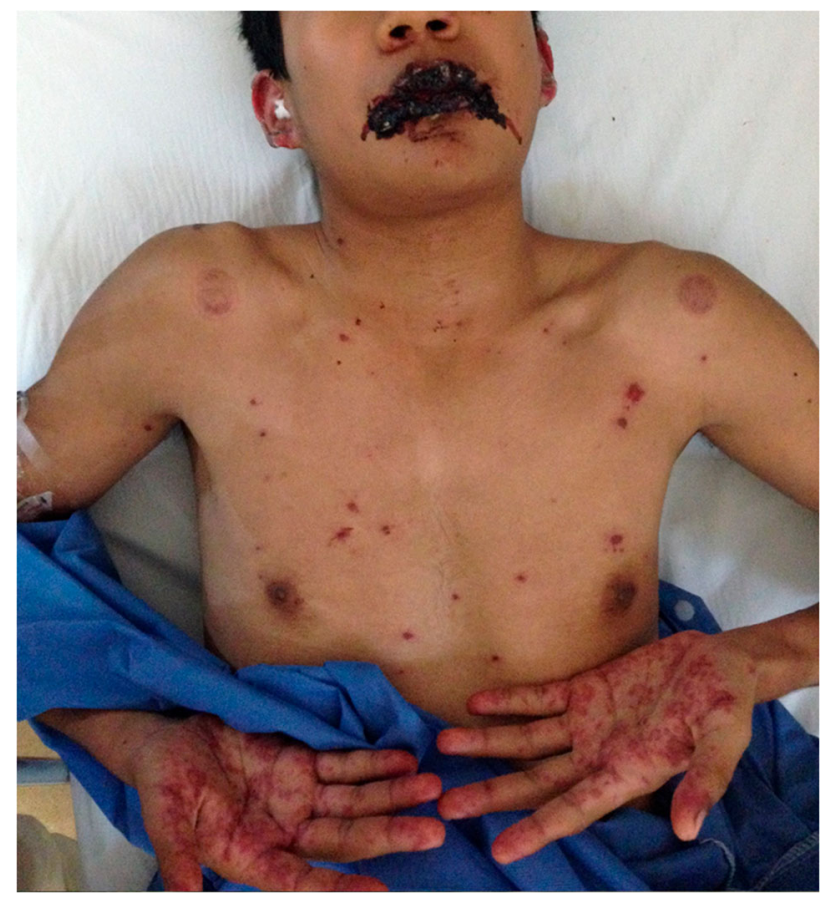

Fig. 1 Polymorphous skin rash and mucositis of the lips. The skin lesions included non-pruritic erythematous lesions, erupted vesicles in chest, targetoid lesions in palms, and areas of denuded skin in ears. The minimal suction generated by the EKG suction electrodes on the skin of shoulders caused erythematous lesions 


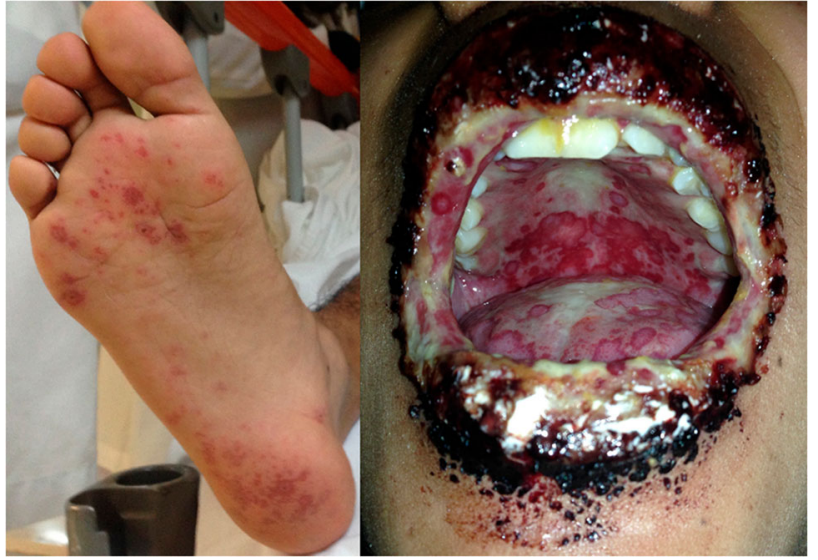

Fig. 2 Feet and mouth manifestations. Targetoid lesions with darker center in foot. Severe mucositis of the lips and the buccal mucosa

Corresponding Author: Aldo Barajas-Ochoa, MD; Department of Medicine, Rutgers New Jersey Medical School, Newark, NJ, USA (e-mail: aldouch5@gmail.com).

\section{Compliance with Ethical Standards:}

Conflict of Interest: Aldo Barajas-Ochoa declares the absence of conflict of interest related to this publication, including personal and financial conflicts.

Nora Montealegre declares the absence of conflict of interest related to this publication, including personal and financial conflicts.

Arturo Perez-Peralta declares the absence of conflict of interest related to this publication, including personal and financial conflicts.

\section{REFERENCES}

1. Lerch M, Mainetti C, Beretta-Piccoli BT, Harr T. Current perspectives on Stevens-Johnson syndrome and toxic epidermal necrolysis. Clinic Rev Allerg Immunol 2018;54:147-176.

2. Alvarado SA, Munoz-Mendoza D, Bahna SL. High-risk drug rashes. Ann Allergy Asthma Immunol 2018;121:552-560.

3. High WA. Stevens-Johnson syndrome and toxic epidermal necrolysis: Pathogenesis, clinical manifestations, and diagnosis. Adkinson NF, Levy ML, Mockenhaupt M, eds. UpToDate. Waltham: UpToDate Inc. https:// www.uptodate.com (Accessed on September 16, 2019.)

4. Morales ME, Purdue GF, Verity SM, Arnoldo BD, Blomquist PH. Ophthalmic manifestations of Stevens-Johnson syndrome and toxic epidermal necrolysis and relation to SCORTEN. Am J Ophthalmol 2010; 150:505-510.

Publisher's Note Springer Nature remains neutral with regard to jurisdictional claims in published maps and institutional affiliations. 